\title{
Gross Local Happiness
}

\author{
To all students, teachers and parents
}

\author{
TSEWANG LHUNDUP
}

\begin{abstract}
The 21st century promises to be the "century of interdependence." Yet, growing interdependence is no guarantee for greater equity of sustainable and just development. To ensure that increasing interdependence leads to an extending and deepening mind, the education system must appropriately respond to and coordinate the complex dynamics that characterize 21st century social, economic, political, and cultural realities. The consensus from the students, teachers, parents, and policymakers throughout Bhutan is clear. Ancient wisdom needs to be integrated into the future Education system. This paper proposes forging a coordinated approach to an educational system systematically informed by traditional conceptual resources, which is consonant with future education systems to 'form the mind' rather than 'filling' it. Such an educational system would organically develop the body, speech, and mind while effectively interacting with people, culture, and environment. In particular, it will secure emotional intelligence and mental balance. That is essential to stay relevant and stay ahead in the age of algorithms.
\end{abstract}

KEYWORDS: Fourth industrial revolution; Interdependence of all things; Pratitya-samutpada; Gross National Happiness; Gross Local Happiness

\section{Imperatives for a New Approach to the educational system}

This Fourth Industrial Revolution is, however, fundamentally different. It is characterized by a range of new technologies that are fusing the physical, digital and biological worlds, impacting all disciplines, economies and industries, and even challenging ideas about what it means to be human, (Schwab, K., 2017). The fourth industrial revolution (First published in Great Britain by Portfolio). Portfolio Penguin. It may even influence our views on what it means to be human. The speed and complexity of the Fourth Industrial Revolution with present-day patterns of globalization are historically unprecedented, bringing about fundamental systems of change in the way we live, work and co-exist that at once open and integrate societies worldwide. The cross-cutting impacts of digital, biological, and physical spheres are even more important than the exciting capabilities they represent. Still, these systems are increasingly not only complicated but also complex. 
Given several thousand years of collective human civilization accumulated data, the behavior of complicated systems can be (within accepted statistical parameters) accurately predicted and managed. By contrast, complex systems exhibit behaviors that, in principle (and not simply in practice) could not be unanticipated. Complex systems generate novel behaviors by their recursive structure, by means of which histories of the situational outcomes of their own behaviors feed-forward into shaping future behavior - if you want new ideas, read old books. Coherently responding to complex systems and change is a unique opportunity to develop a new understanding of what it 'human-centric could mean, and what at a human-centric future education system could look like in the Anthropocene.

\section{Forging a Holistic Educational System Paradigm}

Contrary to the earlier three revolutions, this fourth industrial revolution will not just be one big bang event, where everything settles to a new equilibrium and life continues to revolve around throughout the 21st century. Instead, it will be cascading of even more considerable disruption every decade. To thrive as human beings, we have to evolve all aspects of nature to keep up with the capacities of technology and challenge its dynamism. Predicaments' disruptive force is confronting our meaningful, relevant existence, and it can only be resolved through holistic education - by developing psychological well-being, emotional intelligence, and mental balance. It is now relatively common for various educational systems in many countries to affirm that the driving values often lie outside the current educational system.

In the current field of education, it is presumptive that skills relate to some future experiences. We resource our knowledge needed to survive in society's white and blue color workers. However, perhaps this survival mode agenda is irrelevant because there will be only yes color jobs in the future due to the scientific breakthroughs in fields such as machine learning, robotics, artificial intelligence and Quantum computing. This would be one of the main assumptions that any future education system should challenge.

Overtly, in the last few centuries, we have witnessed how the modern educational system has not been accompanied by a comparable spiritual, intellectual, emotional, and mental advancement. These dimensions of human life have traditionally been safeguarded and transmitted over many thousand years through the monastic education system. However, all the philosophical and spiritual questions have to be asked in a new key in the present age. Philosophers who have debated over several thousand years are now questioning scientists and engineering. The second challenge for any future education system is revitalizing focus on inner values and radically altering the current system.

\section{Monastic Education in Bhutan.}

The monastic education system offers a promising body of resources for addressing complex change and meeting the need for meaningfully contributing to the development of future education systems. This is especially true in Bhutan, where the government has given equal importance to both the Monastic and modern education systems. As a result, both are thriving in ever peaceful co-existence. But much as the concepts and methods of modern education have global relevance, the ideas and practices proper to the monastic education system can be seen as having widespread and particularly timely salience in a world of increasingly complex, meaning-sensitive interdependence. 
The root practices of monastic education aim to develop keen and caring insight into the interdependence of all things to release human beings from suffering, Pratitya-samutpada, as unfolded for example interpreted according to the Tibetan Book of the Dead (Evans-Wentz, 1927). This education system's insight into the nature of interdependence stresses how values - intentions actions (karma) shape and orient the patterns of outcomes and opportunities that structure the dynamics of interdependence. It is the engagement with interdependence as both dynamic and dramatic (or value- and intention-laden) that underlies the particular promises as a body of resources for crafting future education systems in light of complex and predicament-prone realities ahead.

It is the function of such monastic practice to establish and systematically cultivate the values-intention-actions needed to realize wisdom, attentive mastery, and moral clarity to resolve trouble and suffering. In widely accepted Bhutanese terms, wisdom grows out of caring and skilled insight into and engagement with interdependence. Attentive mastery develops as sustained, focused, and yet flexible attunement to situational dynamics. Moral clarity arises with a mature appreciation of the currents of value-intention-action shaping a given situation and how to direct these currents toward liberating interdependence.

Thus far, however, monastic education concepts and teachings have mostly been marshaled only to mitigate the untoward effects of the modern education system. They have not been systematically mobilized to assess existing educational paradigms critically. Nor have they been used innovatively to articulate distinctively future new educational systems paradigms suited to contemporary circumstances.

\section{Gross National Happiness and Gross Local Happiness}

However, significant progress in this direction has been made in Bhutan through the inspired challenge posed by His Majesty, King Jigme Singye Wangchuck, to orient development activities toward increasing Gross National Happiness (Ura, K., \& Centre for Bhutan Studies, 2012). By stating that Gross National Happiness (GNH) will be more important than Gross National Product (GNP), His Majesty explicitly subordinated quantitative to qualitative criteria for policy evaluation and implicitly called for a fully coordinated approach to policy formulation and implementation.

The 5th - five-year development Plan (FYP - 1981-87) anticipated the need for coordinated public policy by departing from the emphasis of prior 5-Year Plans on sector divided objectives to emphasize cross-sectoral aims. In addition, from this time forward, Bhutanese planning has been more visionary in aspiration, stressing the need for proactive policies in keeping with such broad values as sustainability, consolidating and conserving cultural identity, and decentralization. From the 6th FYP (1987-92) onward, GNH has served as an umbrella for identifying emerging, proactive concerns and commitments related to environmental and cultural conservation, good governance, and equitable economic development.

GNH is often described as resting on four pillars: the promotion of environmental conservation, cultural preservation, equitable economic development, and good governance. The broad and encompassing nature of these four developmental domains has the merit of including virtually all development activities under the rubric of GNH.

However, by implicitly focusing on the national level, the term raises immediate questions of categorical relevance and measurement. Given that happiness consists of quality and direction of relationship, the concept of a nation involved in GNH should also be glossed in fully relational terms as a responsive interface merging local with national patterns of relationship. 
Thus what I will call the Gross local Happiness (GLH) concerns radical collaboration at the grassroots level.

It is imperative that GLH be implemented in light of both local and national dynamics. That is, these dynamics must be sensitive to local trends affecting the interplay of different activity domains. In local terms, they should include incisive, deeply historical analyses of the valuesintentions-actions that have been and continue to drive communal Bhutan practices associated with each domain. In some cases, emerging contemporary realities may recommend a shift of previously existing institutional boundaries and associated domains.

Diversity is often misused as a synonym for variety or multiplicity. In ecological terms, diversity measures the resilience of a self-sustaining ecosystem. It is tied directly to the extent and depth of interdependencies by means of which individual species convert situational resources in ways that contribute to one another's welfare. Indeed, individual species are or come to be as a complex function of what they mean to one another. These meaningful interdependencies are the basis on which ecosystems arise and can respond to stress. Ecological diversity is not a function of fundamentally competing species but the density and depth of contributory relationships.

Given that all things have no essential nature, arising in complex interdependence and interpenetration, each thing can be seen as a nexus of contributions from and to all other things. Diversity arises with self-sustaining systems of relationships through which each thing appreciates or draws out the value of their situation as a whole, for the benefit of all others. Each thing ultimately is what it means for all other things. Insofar as all things are empty, they are all ultimately the same. But they are the same precisely because each uniquely expresses the contributory force of all others. Thus, heightened GLH, means heightened GNH in the maximal appreciation of situational resources, relating freely to mutually beneficial contribution to shared welfare.

Heightened GNH and heightened GLH can be seen as crucial indices of increasing personal, communal, and national happiness. Together, they index the tranquility of the "body, speech and mind" and the opening of new and meaningful possibilities for relating freely in the focused and flexibly attentive realization of truly common goods.

\section{Toward a Coordinative wholesome education future Education Paradigm} B

Education recommends itself as a befitting answer to the fourth industrial revolution by crafting "wholesome education" attentive to the needs of students as whole persons.

The following brief comments on future education are offered as a synopsis for the desired future education system.

The globally dominant educational paradigm can be characterized as orienting education toward engendering individual and collective competencies that embody highly context-dependent abilities to take part in reproducing (and incrementally extending) contemporary norms and practices. This paradigm is increasingly misaligned with contemporary realities, as outlined above. These realities practically command a shift toward educational practices suited to engendering virtuosity in improvising context-revising, anticipatory norms, and practices. Such a paradigm would not center on preserving or modestly reforming abilities to fit into current and anticipated social, economic, political and cultural conditions, but rather on 
cultivating the complex sensibilities and skills needed to virtuosically accommodate and direct conditions that could not have been anticipated.

Educational systems aimed at fostering the acquisition of presently relevant skills and knowledge are suitable only in the context of relatively slow and predictable change. Consequently, they are ill-suited for responding to the imperatives and opportunities of rapid change and global diversity. In effect, competency-based education contributes to significant friction between available attentive and responsive resources and changing realities and needs and can introduce considerable drag in the overall pattern of societal development.

The complex and accelerating change characteristic of 21st-century patterns of interdependence suggests the merits of a shift away from a focus on objective knowledge and competencies to an educational paradigm focused on relational maturity and exemplary skills in shared meaning-making. In its broadest terms, monastic education offers a coherent model for undertaking such a paradigm shift.

The central aim of monastic education is the expression of relational virtuosity in resolving suffering. Because suffering announces relational disruption or discord, and because valuesintentions-actions (karma) play a pivotal role in shaping relationships, the liberating virtuosity aimed at in monastic education implies consummate skill in negotiating and revising the meaning of a given situation, for the purpose of enabling all participants to contribute freely to the realization of truly shared welfare. Because of the changing nature of all situations, this skill necessarily implies fully realized excellence in improvisation. In sum, monastic education is not primarily or substantially preparation for problem-solving but training in liberating predicament-resolution.

Virtuosity bridges the traditional (and in the dominant model of education, firmly segregated) categories of knowledge and wisdom. Virtuosity is an expression of situationally specific contributory genius - an utterly fluid and skillfully productive power of live engagement. But more than that, virtuosity connotes a stunningly graceful capacity for dramatic immediacy and innovation. Virtuosity is relating freely within situation-specific limitations to bring about a coordinated appreciation of all that is present.

The means and ends of monastic education, in the very broadest sense, have been wisdom, attentive mastery, and moral clarity. An educational ideal, which aims for caring and effective insight into interdependence, focused yet flexible awareness, and clarity of response in dramatically or morally complex situations, will also lead to a reconfiguration of curricula that takes relationships as primary. That marks a significant shift away from curricula focused on building individual cognitive, emotional, and physical competencies. The "whole person" educated in competency-focused curricula is a person in substantial isolation from others and the demands of complex realities. Indeed, in keeping with the Buddhist teaching of no-self- that is, the irreducible relationality of persons - the identification of independent cognitive, emotional, and physical abilities must be seen as ontologically prejudiced and counterproductive. Curricula focused on relational virtuosity will, at the very least, center on coordinating activities that bring students together in the shared realization of complex ends. They would also introduce students to the full range of activity domains comprising and informing local, national, and global society. These would run the gamut from parenting activities through homemaking, trade work, technical and professional activity, and both secular and sacred leadership. Emphasis would be on revealing interdependencies and hands-on experience.

According to such a paradigm, educational progress will not be measured or determined strictly or even primarily in terms of what is known, but how students are present- bodily, mentally, and emotionally. The more highly educated persons and communities become, the more readily, relevantly, and responsibly they will be able and inclined to demonstrate truly 
appreciative and contributory virtuosity. Education can be measured, then, in terms of personally and communally achieved and sustained happiness in the relational terms outlined above. The particulars of effecting such a shift of educational paradigms must vary from locale to locale. Moreover, such a shift is not a one-time event but rather the initiation of commitment to a particular trajectory of the ongoing and ever-expanding fourth industrial revolution.

Education practices that cultivate appreciative and contributory virtuosity need not eschew science and technology any more than they need to be in tension with the conservation of culture and traditions. But such practices would radically reframe priorities within curricula to reflect a commitment to promoting mindful and energetic attunement to relationships and interdependencies in such a way that student (as well as teacher, parent, and social) stress is reduced. Only on the basis of tranquil students and student bodies will they be able to go beyond cooperation in a spirit of competition to true coordination in concentrated attention to the possibilities for infusing all local, national and global environments with equanimity, compassion, loving-kindness, and joy in the good fortune of others.

Education practiced along these lines will change the way all social, economic, political, cultural, and environmental activity is undertaken and understood. It would play a crucial part in driving all fourth industrial revolution activities toward engendering greater happiness in all the complex interdependencies linking the personal, the communal, the national, and the global.

"There is no way to happiness; happiness is the way" - The Buddha.

\section{Literature}

Evans-Wentz, W. Y. (1927). The Tibetan book of the dead. Oxford: Oxford University Press. Schwab, K. (2017). The fourth industrial revolution (First published in Great Britain by Portfolio). Portfolio Penguin.

Ura, K., \& Centre for Bhutan Studies. (2012). An extensive analysis of GNH index. Thimphu, Bhutan: Centre for Bhutan Studies. 